Initiative Program for Digitalization of Photographic Plate Archives at the Central Research Institute of Film Materials and Artificial Leathers $\left[1960^{\text {th }}-1980^{\text {th }}\right.$ ] Polymer Chemistry

Part I. Alphabetic list [Cyrillic]

\title{
AБC
}

1. Multiparametric Microscopy Facility Subdivision: OCR station, "ПВХ - АБC 7-3", IEEE Dataport, 2019. [Online]. Available: http://dx.doi.org/10.21227/tccz-wn91. Accessed: Dec. 30, 2019.

\section{ДСТ -30}

1. Multiparametric Microscopy Facility Subdivision: OCR station, "ДСТ-30 ПОСЛЕ ОБРАБОТКИ ЧЕТЫРЕХОКИСЬЮ ОСМИЯ [Х 39 000]", IEEE Dataport, 2019. [Online]. Available: http://dx.doi.org/10.21227/20a5-4f90. Accessed: Dec. 30, 2019.

2. Multiparametric Microscopy Facility Subdivision: OCR station, "ДСТ-30 [x 82 000]", IEEE Dataport, 2019.

[Online]. Available: http://dx.doi.org/10.21227/4 zmb-4321. Accessed: Dec. 30, 2019.

3. Multiparametric Microscopy Facility Subdivision: OCR station, "ДСТ-30+ ПН-6 Х30000", IEEЕ Dataport, 2019.

[Online]. Available: http://dx.doi.org/10.21227/2r5b-da89. Accessed: Dec. 30, 2019.

4. Multiparametric Microscopy Facility Subdivision: OCR station, "ДСТ-30 +TРМ X30000", IEEE Dataport, 2019.

[Online]. Available: http://dx.doi.org/10.21227/2m8q-y269. Accessed: Dec. 30, 2019.

5. Multiparametric Microscopy Facility Subdivision: OCR station, "ДСТ-30, 40, 50 ИСТ", IЕEЕ Dataport, 2019.

[Online]. Available: http://dx.doi.org/10.21227/ggz0-8p67. Accessed: Jul. 24, 2020.

\section{HK}

1. Multiparametric Microscopy Facility Subdivision: OCR station, "HK + CKH 40 + CAЖA", IEEE Dataport, 2019. [Online]. Available: http://dx.doi.org/10.21227/8w61-1h45. Accessed: Dec. 30, 2019.

\section{$\Pi \mathrm{A}$}


1. Multiparametric Microscopy Facility Subdivision: OCR station, "ПЭ + ПA", IEEE Dataport, 2019. [Online].

Available: http://dx.doi.org/10.21227/csdy-s728. Accessed: Dec. 30, 2019.

2. Multiparametric Microscopy Facility Subdivision: OCR station, "15 ПЭ + ПA", IEEE Dataport, 2019. [Online]. Available: http://dx.doi.org/10.21227/289t-q822. Accessed: Dec. 30, 2019.

3. Multiparametric Microscopy Facility Subdivision: OCR station, "ПЭ + ПА + MEЛ", IEEE Dataport, 2019. [Online]. Available: http://dx.doi.org/10.21227/k7v9-pm48. Accessed: Dec. 30, 2019.

\section{ПBX}

1. Multiparametric Microscopy Facility Subdivision: OCR station, "ПВХ - АБС 7-3", IEEE Dataport, 2019. [Online]. Available: http://dx.doi.org/10.21227/tccz-wn91. Accessed: Dec. 30, 2019.

2. Multiparametric Microscopy Facility Subdivision: OCR station, "ПС-ПВХ СМЕСЬ 50-50 иЗ ДХЭ", IEЕE Dataport, 2019. [Online]. Available: http://dx.doi.org/10.21227/v8rc-0j93. Accessed: Dec. 30, 2019.

3. Multiparametric Microscopy Facility Subdivision: OCR station, "ПС-ПВХ, СМЕСЬ 20-80 ХБ", IEEE Dataport, 2019.

[Online]. Available: http://dx.doi.org/10.21227/dk35-n953. Accessed: Dec. 30, 2019.

\section{ПИБ-200}

1. Multiparametric Microscopy Facility Subdivision: OCR station, "ПИБ-200", IЕEЕ Dataport, 2019. [Online]. Available: http://dx.doi.org/10.21227/ce8p-xa83. Accessed: Dec. 30, 2019.

2. Multiparametric Microscopy Facility Subdivision: OCR station, "ПИБ-200 + САЖА АЦЕТИЛЛЕНОВАЯ", IЕEЕ Dataport, 2019. [Online]. Available: http://dx.doi.org/10.21227/3h6n7n71. Accessed: Dec. 30, 2019.

3. Multiparametric Microscopy Facility Subdivision: OCR station, "ПИБ-200 СТЕКЛО X 10400", IEEЕ Dataport, 2019. [Online]. Available: http://dx.doi.org/10.21227/1c42-w681. Accessed: Dec. 30, 2019.

4. Multiparametric Microscopy Facility Subdivision: OCR station, "ПИБ-200 СТЕКЛО X 18200", IEEЕ Dataport, 2019. [Online]. Available: http://dx.doi.org/10.21227/5fcb-6d49. Accessed: Dec. 30, 2019.

\section{$\Pi \mathrm{H}$}

1. Multiparametric Microscopy Facility Subdivision: OCR station, "ДСТ-30+ ПН-6 X30000", IEЕЕ Dataport, 2019. [Online]. Available: http://dx.doi.org/10.21227/2r5b-da89. Accessed: Dec. 30, 2019. 


\section{ПMMA}

1. Multiparametric Microscopy Facility Subdivision: OCR station, "ПС - ПMМA", IЕEЕ Dataport, 2019. [Online]. Available: http://dx.doi.org/10.21227/cc5j-k722. Accessed: Dec. 30, 2019.

2. Multiparametric Microscopy Facility Subdivision: OCR station, "ПC + ПMMA [x 10 400]", IEEE Dataport, 2019. [Online]. Available: http://dx.doi.org/10.21227/6q4c-0902. Accessed: Dec. 30, 2019.

\section{ППУ}

1. Multiparametric Microscopy Facility Subdivision: OCR station, "СКН-40 + ПЕНОПОЛИУРЕТАН", IEЕЕ Dataport, 2019. [Online]. Available: http://dx.doi.org/10.21227/qsa8-kh65. Accessed: Dec. 30, 2019.

\section{$\Pi \mathrm{C}$}

1. Multiparametric Microscopy Facility Subdivision: OCR station, "ПС - ПMMA", IEEE Dataport, 2019. [Online]. Available: http://dx.doi.org/10.21227/cc5j-k722. Accessed: Dec. 30, 2019.

2. Multiparametric Microscopy Facility Subdivision: OCR station, "ПC + ПMMA [x 10 400]", IEEE Dataport, 2019. [Online]. Available: http://dx.doi.org/10.21227/6q4c-0902. Accessed: Dec. 30, 2019.

3. Multiparametric Microscopy Facility Subdivision: OCR station, "ПС-ПВХ, СМЕСЬ 20-80 ХБ", IEEE Dataport, 2019. [Online]. Available: http://dx.doi.org/10.21227/dk35-n953. Accessed: Dec. 30, 2019.

\section{ПСТ}

1. Multiparametric Microscopy Facility Subdivision: OCR station, "ПСТ - СКС 85 [8000X]", IEEE Dataport, 2019. [Online]. Available: http://dx.doi.org/10.21227/nkbe-mz75. Accessed: Dec. 30, 2019.

\section{ПTXБ}

1. Multiparametric Microscopy Facility Subdivision: OCR station, "ПТХБ + СКД (7/3) X $10000 "$, IEEE Dataport, 2019. [Online]. Available: http://dx.doi.org/10.21227/jsvg-sk45. Accessed: Dec. 30, 2019.

\section{$\Pi \ni$}

1. Multiparametric Microscopy Facility Subdivision: OCR station, "ПОЛИЭТИЛЕН + МЕЛ", IЕEЕ Dataport, 2019. [Online]. Available: http://dx.doi.org/10.21227/dzsr-yb84. Accessed: Dec. 30, 2019. 
2. Multiparametric Microscopy Facility Subdivision: OCR station, "ПЭ + ПА + MEЛ", IEEE Dataport, 2019. [Online]. Available: http://dx.doi.org/10.21227/k7v9-pm48. Accessed: Dec. 30, 2019.

3. Multiparametric Microscopy Facility Subdivision: OCR station, "ПЭ + ПA", IEEE Dataport, 2019. [Online]. Available: http://dx.doi.org/10.21227/csdy-s728. Accessed: Dec. 30, 2019.

\section{СИH -40}

1. Multiparametric Microscopy Facility Subdivision: OCR station, "Гели СИН-40", IEEE Dataport, 2019. [Online]. Available: http://dx.doi.org/10.21227/f4cc-af46. Accessed: Dec. 30, 2019.

\section{СКД}

1. Multiparametric Microscopy Facility Subdivision: OCR station, "ПТХБ + СКД (7/3) X $10000 "$, IEEE Dataport, 2019. [Online]. Available: http://dx.doi.org/10.21227/jsvg-sk45. Accessed: Dec. 30, 2019.

2. Multiparametric Microscopy Facility Subdivision: OCR station, "СКД-СКН-18 3/1", IEEE Dataport, 2019. [Online]. Available: http://dx.doi.org/10.21227/abaq-te56. Accessed: Dec. 30, 2019.

3. Multiparametric Microscopy Facility Subdivision: OCR station, "СКД - СКН-18 1-3 [x5050]", IEEE Dataport, 2019. [Online]. Available: http://dx.doi.org/10.21227/178b-bj40. Accessed: Dec. 30, 2019.

\section{CKH -18}

1. Multiparametric Microscopy Facility Subdivision: OCR station, "CKH-18 [x 5050]", IEEE Dataport, 2019. [Online]. Available: http://dx.doi.org/10.21227/esv7-zf42. Accessed: Dec. 30, 2019.

2. Multiparametric Microscopy Facility Subdivision: OCR station, "СКД - СКН-18 1-3 [x5050]", IEEE Dataport, 2019. [Online]. Available: http://dx.doi.org/10.21227/178b-bj40. Accessed: Dec. 30, 2019.

3. Multiparametric Microscopy Facility Subdivision: OCR station, "СКД-СКН-18 3/1", IEEE Dataport, 2019. [Online]. Available: http://dx.doi.org/10.21227/abaq-te56. Accessed: Dec. 30, 2019 .

\section{CKH -40}

1. Multiparametric Microscopy Facility Subdivision: OCR station, "СКН-40-СКД 1-1", IEEE Dataport, 2019. [Online]. Available: http://dx.doi.org/10.21227/tdqe-rq16. Accessed: Dec. 30, 2019.

2. Multiparametric Microscopy Facility Subdivision: OCR station, "СКН-40 + ТЕРМИЧЕСКАЯ САЖА", IEЕE Dataport, 2019. 
[Online]. Available: http://dx.doi.org/10.21227/7z80-4p49. Accessed: Dec. 30, 2019.

3. Multiparametric Microscopy Facility Subdivision: OCR station, "HK + CKH 40 + CAЖA", IEEE Dataport, 2019.

[Online]. Available: http://dx.doi.org/10.21227/8w61-1h45. Accessed: Dec. 30, 2019.

4. Multiparametric Microscopy Facility Subdivision: OCR station, "СКН-40 + СКЭПТ-50 (70/50)", IEEE Dataport, 2019. [Online]. Available: http://dx.doi.org/10.21227/ty4r-dx42. Accessed: Dec. 30, 2019.

5. Multiparametric Microscopy Facility Subdivision: OCR station, "СКН-40 + ПЕНОПОЛИУРЕтАН", IEEЕ Dataport, 2019.

[Online]. Available: http://dx.doi.org/10.21227/qsa8-kh65. Accessed: Dec. 30, 2019.

\section{CKC}

2. Multiparametric Microscopy Facility Subdivision: OCR station, "ПСТ - СКС 85 [8000X]", IEEE Dataport, 2019. [Online]. Available: http://dx.doi.org/10.21227/nkbe-mz75. Accessed: Dec. 30, 2019.

\section{СКЭПТ}

1. Multiparametric Microscopy Facility Subdivision: OCR station, "СКН-40 + СКЭПТ-50 (70/50)", IEEE Dataport, 2019. [Online]. Available: http://dx.doi.org/10.21227/ty4r-dx42. Accessed: Dec. 30, 2019.

\section{СПЛ}

1. Multiparametric Microscopy Facility Subdivision: OCR station, "СПЛ-1", IEEE Dataport, 2019. [Online]. Available: http://dx.doi.org/10.21227/q2q9-7r16. Accessed: Dec. 30, 2019.

2. Multiparametric Microscopy Facility Subdivision: OCR station, "СПЛ II", IEEE Dataport, 2019. [Online]. Available: http://dx.doi.org/10.21227/swt0-s979. Accessed: Dec. 30, 2019.

3. Multiparametric Microscopy Facility Subdivision: OCR station, "CПЛ V", IEEE Dataport, 2019. [Online]. Available: http://dx.doi.org/10.21227/cq2w-nh51. Accessed: Dec. 30, 2019.

4. Multiparametric Microscopy Facility Subdivision: OCR station, "Дисперсия СПЛ и дисперсия отходов", ІЕЕЕ Dataport, 2019. [Online]. Available: http://dx.doi.org/10.21227/7yep-0f45. Accessed: Dec. 30, 2019.

5. Multiparametric Microscopy Facility Subdivision: OCR station, "СПЛ (конц. дисперсия - генерация ансамблей)", IEEE Dataport, 2019. [Online]. Available: http://dx.doi.org/10.21227/48fp-fn86. Accessed: Dec. 30, 2019 . 
6. Multiparametric Microscopy Facility Subdivision: OCR station, "СПЛ - насыщенная дисперсия, принудительный контакт без агрегации", IEEE Dataport, 2019. [Online]. Available: http://dx.doi.org/10.21227/xe6q-nx44. Accessed: Dec. 30, 2019.

\section{TPM}

1. Multiparametric Microscopy Facility Subdivision: OCR station, "ДСТ-30 +TPM X30000", IEEE Dataport, 2019.

[Online]. Available: http://dx.doi.org/10.21227/2m8q-y269. Accessed: Dec. 30, 2019.

\section{СКД-CKH}

1. Multiparametric Microscopy Facility Subdivision: OCR station, "СКД-СКН-18 3/1", IEEE Dataport, 2019. [Online]. Available: http://dx.doi.org/10.21227/abaq-te56. Accessed: Dec. 30, 2019.

2. Multiparametric Microscopy Facility Subdivision: OCR station, "СКД - СКН-18 1-3 [x5050]", IEEE Dataport, 2019. [Online]. Available: http://dx.doi.org/10.21227/178b-bj 40 . Accessed: Dec. 30, 2019.

3. Multiparametric Microscopy Facility Subdivision: OCR station, "СКН-40-СКД 1-1", IEEE Dataport, 2019. [Online]. Available: http://dx.doi.org/10.21227/tdqe-rq16. Accessed: Dec. 30, 2019 .

\section{Composites and Compositions}

Singular Examples

\section{$\Pi C-\Pi B X$}

1. Multiparametric Microscopy Facility Subdivision: OCR station, "ПС-ПВХ СМЕСЬ 50-50 ИЗ ДХЭ", IEЕЕ Dataport, 2019. [Online]. Available: http://dx.doi.org/10.21227/v8rc-0j93. Accessed: Dec. 30, 2019.

2. Multiparametric Microscopy Facility Subdivision: OCR station, "ПС-ПВХ, СМЕСЬ 20-80 ХБ", IЕEЕ Dataport, 2019. [Online]. Available: http://dx.doi.org/10.21227/dk35-n953. Accessed: Dec. 30, 2019.

\section{ПС-ПMМА}

1. Multiparametric Microscopy Facility Subdivision: OCR station, "ПС - ПMMA", IEEE Dataport, 2019. [Online]. Available: http://dx.doi.org/10.21227/cc5j-k722. Accessed: Dec. 30, 2019.

2. Multiparametric Microscopy Facility Subdivision: OCR station, "ПC + ПMMA [x 10 400]", IEEE Dataport, 2019. [Online]. Available: http://dx.doi.org/10.21227/6q4c-0902. Accessed: Dec. 30, 2019. 


\section{Glass and Plastic}

1. Multiparametric Microscopy Facility Subdivision: OCR station, "PLASTIC X ON THE GLASS", IEEE Dataport, 2019.

[Online]. Available: http://dx.doi.org/10.21227/txs9-8r45. Accessed: Dec. 30, 2019.

2. Multiparametric Microscopy Facility Subdivision: OCR station, "ПИБ-200 СТЕКЛО X 10400", IEEE Dataport, 2019. [Online]. Available: http://dx.doi.org/10.21227/1c42-w681. Accessed: Dec. 30, 2019.

3. Multiparametric Microscopy Facility Subdivision: OCR station, "ПИБ-200 СТЕКЛО X 18200", IЕEЕ Dataport, 2019. [Online]. Available: http://dx.doi.org/10.21227/5fcb-6d49. Accessed: Dec. 30, 2019.

\section{Fibers}

1. Multiparametric Microscopy Facility Subdivision: OCR station, "КОМПОЗИТНЫЕ ВОЛОКНА ПЭ ПЭТФ СРЕЗЫ", ІЕЕЕ Dataport, 2019. [Online]. Available: http://dx.doi.org/10.21227/yjc6-wj89. Accessed: Dec. 30, 2019 .

Fig 1:

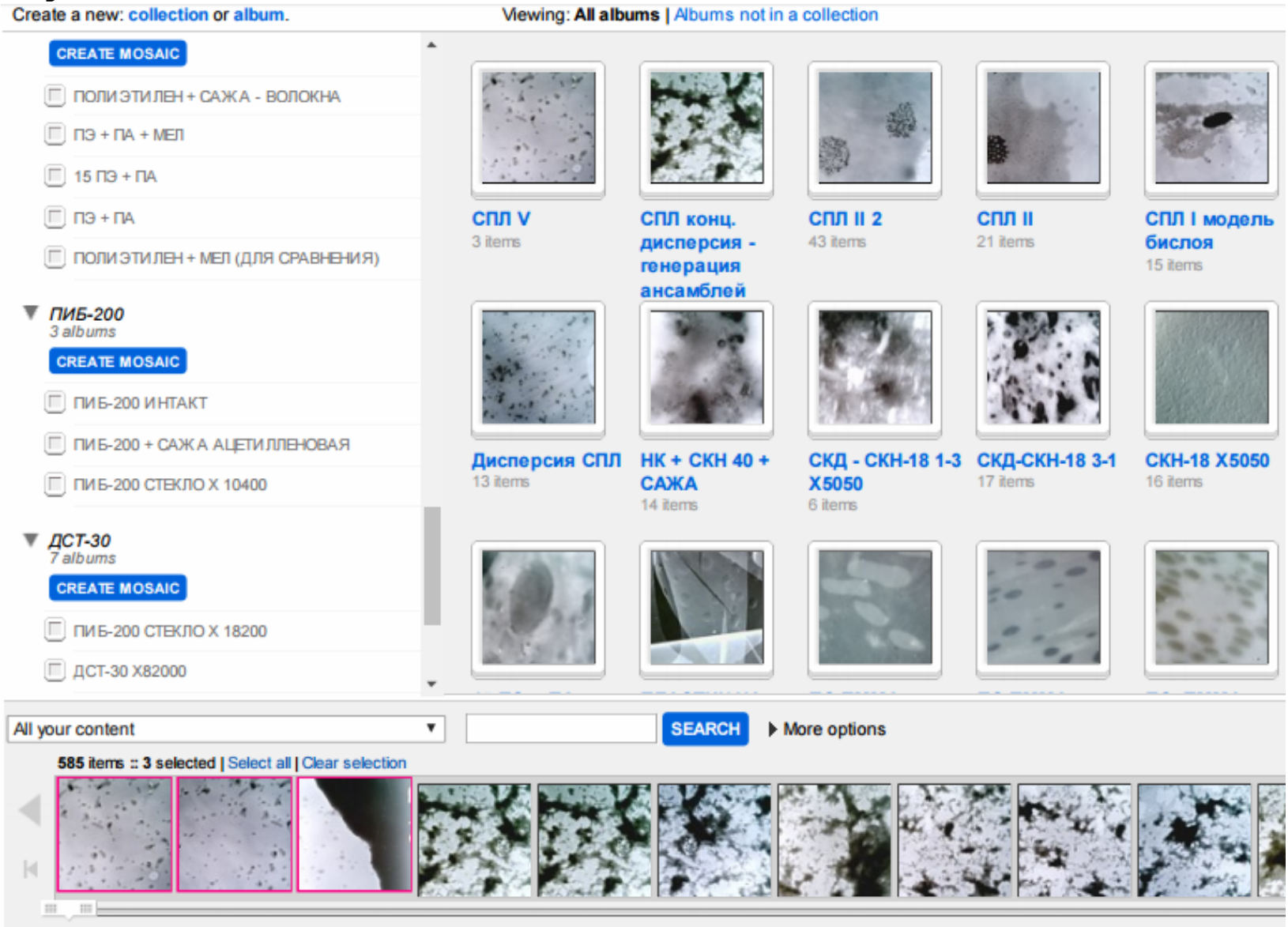




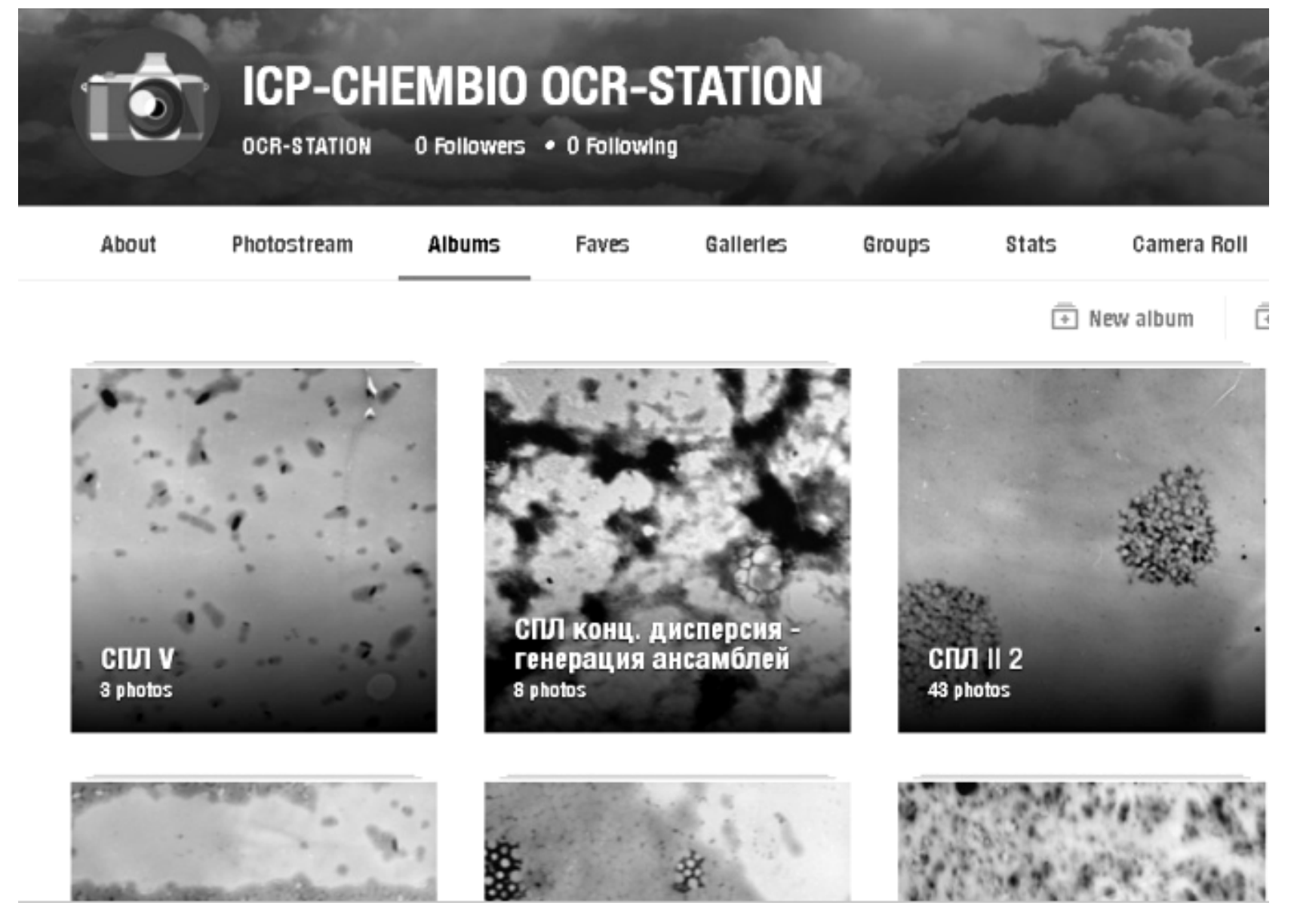

